\title{
On the Coverage Problem in Video-based Wireless Sensor Networks
}

\author{
Stanislava Soro and Wendi B. Heinzelman \\ Department of Electrical and Computer Engineering \\ University of Rochester \\ Rochester NY, 14627 \\ Email: \{soro,wheinzel\}@ece.rochester.edu
}

\begin{abstract}
Video-based wireless sensor networks continue to gain increasing interest due to their ability to collect visual information for a wide range of applications. However, knowledge about these types of networks is mostly related to visual algorithms, leaving the networking perspective aside. In this work, we analyze how an algorithm designed for traditional wireless sensor networks, which integrates the coverage and routing problem, behaves in video-based networks. Our results show that because of the unique way that cameras capture data, the sensor network algorithm does not give the expected results in terms of coverage preservation of monitored areas. We discuss the main differences between traditional wireless sensor networks and video-based networks that lead to such a result, and we provide ideas for how protocols should be designed for the unique features of video-based networks.
\end{abstract}

\section{Introduction}

In recent times, there has been increased interest in video surveillance and monitoring applications. The reasons for this interest are diverse, ranging from security demands and military applications to scientific purposes. In the near future it is expected that video-based wireless sensor networks will make these demands become reality. Videobased sensor networks are initially devised as a collection of small, inexpensive, battery operated nodes with the ability to communicate with each other wirelessly over a limited transmission range. What makes these networks different from traditional wireless sensor networks is the fact that the nodes are additionally equipped with very low power cameras. These camera-nodes have the ability to capture images of observed areas at variable rates, to process the data on-board and to transmit the captured data to the user/main processing center.

As an area with potentially many applications, videobased sensor networks impose many new challenges for research. Possibly one of the biggest challenges is how to manage the huge amount of data generated by the cameras. Compared to traditional wireless sensor networks, the amount of data collected by camera-nodes is very large, and routing this raw data is an inefficient use of the network's resources, such as energy and bandwidth. On the other hand, usually not all information is important for the particular application, so the vast majority of the data can be filtered locally. The images taken by the cameras usually contain a high degree of correlation, which can help to reduce the total amount of data in the system and to build a more fault-tolerant system. This filtering out of data should be done locally, which imposes the need for enough processing capabilities on the nodes so that they can send only relevant data for the specific task. Because of the nature of the collected data, it is expected that algorithms for data processing are overall more complex and power demanding than those for other types of sensors.

It is realistic to expect that the high degree of correlation among the cameras' data can be exploited by making a more "intelligent" system, where the functionality of the system will be achieved through coordinated collaboration among the devices. The camera-nodes should be aware of each other and able to handle the data from other nodes in a coordinated manner. Also, in systems for real-time monitoring applications, the delay constraints are strict.

Video-based sensor networks possess several distinctive features that make them different from traditional wireless sensor networks. In this work we point out and explain the important differences between traditional wireless sensor networks and video-based sensor networks. In particular, we show that when wireless sensor networks are additionally equipped with video cameras, the processes of data gathering, data routing and coverage preservation are essentially changed compared to the same processes in wireless sensor networks. This change comes from the way the camera gathers the data, and also from the nature of the data - images that have been collected by the network over space and time.

In traditional wireless sensors networks, the sensor nodes collect information about different phenomena (temperature, concentration of a substance, light intensity, pressure, humidity, etc.) around them, from the area determined by the sensing range of the node. However, video cameras have the unique feature of capturing images of objects/parts of a region that are not necessarily in the cameras' vicinity. The objects covered by the camera can be at arbitrary locations, perhaps distant from the camera. There are some other unique characteristics of the way that cameras capture data. For example, the information content of images taken by different cameras in a system is unique for each of 
them. This is a result of the different relative positions and orientations of the cameras toward the observed objects, resulting in every camera seeing the objects from a unique perspective. In video-based sensor networks, the sensing range of sensor nodes is replaced with the camera's field of view (FoV). The field of view [2] is defined as the maximum volume visible from the camera. The camera therefore is able to capture images of distant areas and objects that appear within the camera's depth of field, which is the distance between the nearest and the farthest object that the camera can capture sharply.

The non-coincidence between the position of each camera and the location of their captured objects/areas motivates us to revise the concept of coverage and energyefficient routing of collected data, which is taken from traditional wireless sensor networks, for video-based sensor networks.

One of the substantial requirements that has to be fulfilled by any system for video surveillance and monitoring is the demand for persistent coverage of certain monitored areas. We are assuming that in the near future, the rapid progress in technology will lead to the production of affordable, low-power video cameras that will not greatly impact the total cost of video-based sensor networks. However, it is expected that efficient energy consumption in wireless sensor networks will be a persistent problem for a longer time. Also, we can imagine that processing of image data locally on the node will require a significant amount of energy that cannot easily be neglected in the system design. In this light, we want to explore how the existing coverage protocols behave when they are adapted for video-based wireless sensor networks.

\section{Telepresence Application for Video-based Wireless Sensor Networks}

Among the many applications for video-based networks, interest in telepresence applications has grown rapidly in recent times. A telepresence system is a system that enables the user to experience being fully present at a physically remote real world site [17]. For example, the goal of the multidisciplinary project named "Being There" at the University of Rochester is to develop a telepresence system that will enable a user to virtually visit some public area, for example a museum, a gallery, etc.

The "Being There" telepresence system is initially designed as a network of wireless nodes equipped with very low power cameras. The camera-nodes are mounted at random locations in the monitored room. All cameras are identical and static, without the possibility of pan, tilt and zoom. Each camera monitors a finite part of the scene, and the cameras' FoVs can overlap, so that images taken from different cameras can be integrated into a complete global view of the scene. Using a control console, a user can navigate and virtually "move" around in the monitored space. The user expresses a willingness to see different parts of the monitored area in the form of a request, which is sent to the main processing center of the network. For every part of the scene, the request contains the coordinates of the "user request window" - a part of the scene that is requested by the user. At the beginning of the network's operations, the main coordinating center obtains the information about which cameras cover particular parts of the monitored scene.

The development of this telepresence system will proceed in several phases. In the early phase of system development, our goal is to find out how existing protocols for sensor networks, initially considering coverage protocols, can be used for this specific application.

However, for this application, a three-dimensional coverage of space is required. This problem is extremely hard to analyze, and some pioneering work has been done in this direction [18]. In order to simplify this problem, initially we assume the task of floorplan monitoring - monitoring of a scene in one plane. In this task, all camera nodes are mounted in one plane (at the ceiling of the monitored room, for example), and they shoot the images of the scene from a parallel plane, as shown in Figure 1.

We assume that in the first phase of system operations, all cameras with overlapped FoVs are jointly calibrated [4], [9]. Because cameras monitor the scene, which is in one plane, we simplify the problem of volumetric coverage, and consider the coverage of the scene that lays on the parallel plane $\pi_{1}$. We assume that all cameras are directed toward this plane, and that the FoV of every camera intersects with the plane $\pi_{1}$. Therefore, we can consider that plane $\pi_{1}$ is covered if all points of this plane are covered by the intersection of at least one camera's FoV and plane $\pi_{1}$.

Because of the unplanned positions of the cameras, the cameras have overlapped FoVs, and the overall degree of coverage varies. For the application, it is good to have all parts of the monitored area covered with at least one camera. While cameras can have overlapped FoVs, and therefore share scene information, the physical positions of the cameras with overlapped FoVs on mounting plane $\pi$ do not necessarily have to be close, as illustrated in Figure 1.

For such a design space, in this work we analyze how an application-aware routing protocol, primary designed for wireless sensor networks, whose goal is to maintain the network's coverage for longer time, behaves when used in video-based wireless sensor networks. This paper is motivated by the work proposed in [1] and presents some interesting simulation results obtained when the protocol for management of active nodes in traditional wireless sensor networks presented in [1] is adapted to a camera-network scenario. The results indicate the necessity for deeper analysis and understanding of coverage preserving and routing problems when dealing with networked cameras.

\section{Related Work}

\section{III.A Overview of video-based sensor networks}

Video-based wireless sensor networks have started to be an active research area for the last few years. Because the 


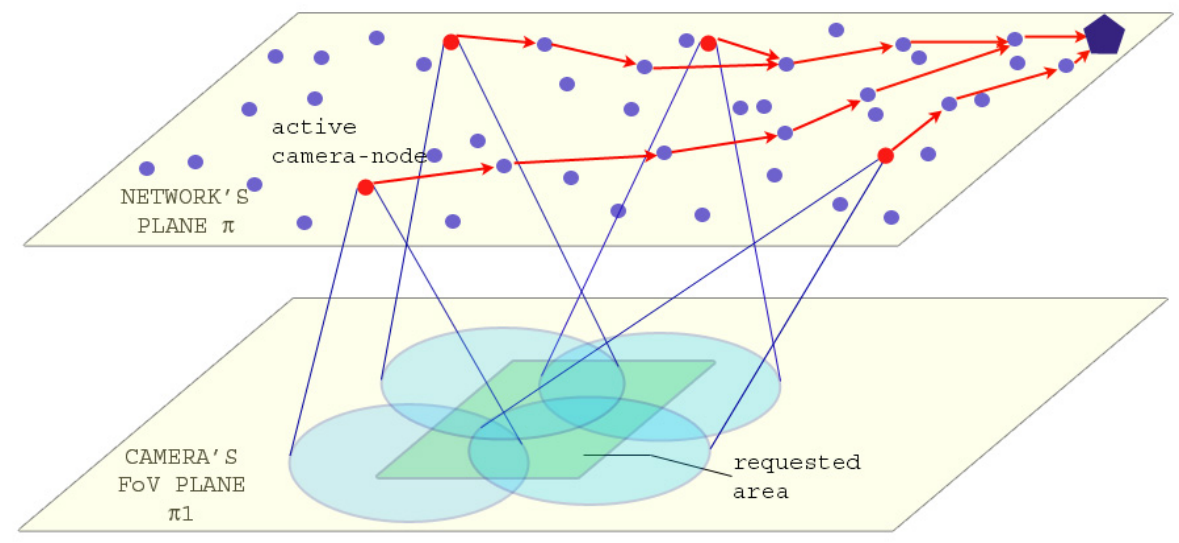

Figure 1 Video-based wireless network and the monitored area. computer vision research area has experienced rapid development in recent time, research on video-based sensor networks has mainly focused on the visual aspect of the network, namely algorithms for image data extraction and analysis. Very little research has been done in order to integrate the knowledge from the vision area and wireless networking for these kinds of systems.

One of the first works that shows the implementation of video cameras for a specific application is described in [3], where video cameras are networked together in order to fulfill the task of collecting interesting oceanographic events. The cameras utilize an automated sampling scheme based on a remote user's demands in order to reduce the need for extremely high bandwidth.

The authors in [4] address the research challenges in video-based sensor networks. In particular, they highlight the importance of sensor collaboration and the development of video-sensor specific network-layer and MAC-layer protocols.

The work in [5] presents the architecture and performance of the Panoptes video-based networking system. The authors show the hardware implementation of this system together with power measurements of system components, as well as developed algorithms for data management. The components are networked in a single hop communication system, and IEEE 802.11 is used as the MAC protocol.

The work presented in [6] proposes a multi-tier, multimodal concept of networks, where the network can be organized into several tiers of cameras with different power and resolution characteristics, and the whole system supports a variety of jointly collaborated sensors, such as vibration, thermal-imaging and camera sensors. The authors claim that the mix of high-performance, high-cost sensors with lowperformance, low-cost sensors can achieve the right balance between system cost and functionality.

Finally, in [7] the authors describe a distributed attention mechanism, which allows the camera-nodes to handle complex situations by focusing on interesting events only and locally discarding uninteresting events.

Many works mentioned here give detailed overviews of ongoing video-networking projects. However, not much attention has been given to the traditional problems of wireless networks, such as scalability, energy-efficient routing of collected data and coverage preservation. We believe that knowledge from sensor networking has to be considered as a base for building efficient video-based networks.

\section{III.B Overview of the coverage problem in sensor networks}

In the past few years, there have been a number of proposed algorithms for coverage preservation in wireless sensor networks. The most commonly used approach to handle this problem is to determine the redundant nodes and put them to sleep. [10] presents a probe-based algorithm, which controls the sleeping of the nodes in order to ensure long lasting coverage. [11] presents work that addresses the problem of selecting the minimum energy-cost connected sensor cover, when each sensor can manage a variable sensing and transmission range. [12] deals with the problem of asymptotic coverage and connectivity, and shows the necessary conditions required such that a specific region is covered with high probability. [13] presents a protocol that dynamically configures the network in order to provide different coverage degrees requested by the application while maintaining connectivity.

In addition to wireless sensor networks, the coverage problem has been researched in other disciplines as well. The well known "art gallery problem" is an example, where the number and the positions of cameras in a gallery room should be determined so that every point is covered by at least one camera. This problem can be solved optimally in a 2-D plane, but becomes NP-hard when it is extended to 3-D space [15].

Most of the mentioned works, however, do not focus on the routing perspective when dealing with the coverage problem. The protocol described in [1] is among the first that integrates the routing and coverage problems together, and exploits how the selection of additional routing nodes impacts the coverage performance of the wireless sensor network.

\section{Application-aware Routing in Wireless Sensor Networks}

The sensor nodes are usually deployed randomly over the monitored area, leading to non-uniform coverage of the monitored area. In order to prevent energy waste, most 
coverage preserving algorithms employ a scheme by which a sufficient number of nodes are powered on in order to cover the given area, while others are put to sleep. Every sensor node chooses one of a few possible states at given moment: to sense the environment, to relay the information toward the sink or to sleep. By managing the appropriate roles of every sensor over time, the coverage of the network can be preserved for a longer period. For example, depending on their position in the network as well as their remaining energy, sensors can have different importance as sensing nodes or relaying nodes. For example, nodes that provide unique coverage of some part are very important for the sensing application, and therefore should be avoided as routing nodes.

Among the many coverage-preserving protocols proposed so far, the DAPR protocol described in [1] is the first known protocol that strives to preserve the coverage of the monitored area while avoiding the routing of data through "critical" nodes. Here "critical" nodes are nodes with higher importance among the others for the sensing application, such as nodes in sparsely deployed areas. On average, the nodes in sparsely deployed areas are more frequently powered on, and they spend their energy mostly on sensing data, so their exploitation as routers for others nodes' data should be avoided. However, traditional energy-aware routing protocols do not take into account this fact, and the decision of whether a particular node should be elected as a routing station solely depends on the energy level at the node. In order to avoid the usage of these critical sensing nodes as routers in the early phase of the network lifetime, [1] proposes an applicationaware routing approach, where every node's importance to the sensing application is evaluated, and this importance is quantified through an application-aware routing cost.

In order to compare and point out the differences between traditional wireless sensor networks and wireless video-based sensor networks with respect to their applicationspecific task (i.e., coverage preservation), we give an outline of the work done in [1], and we show how the DAPR protocol for integrated coverage preservation and routing works in video-based sensor networks.

\section{IV.A Overview of the DAPR protocol}

We assume a scenario where a certain number of sensor nodes are deployed randomly over a rectangular area $A$. The set of deployed nodes is denoted as $S=\left\{S_{1} \ldots S_{n}\right\}$. For the sake of simplicity, we assume that all sensor nodes collect data within the same sensing range. It is assumed that a network has to accomplish an application-specific task, which requires that every point of the monitored area be covered by at least one active sensor.

The random and dense deployment of sensor nodes creates redundancy in the collected information and introduces non-efficient spending of energy. This waste of energy can be eliminated by putting to sleep any unnecessary nodes, while a group of active nodes performs the task of data gathering. Every point $(x, y)$ of the monitored area can be covered by an arbitrary number of sensor nodes. Let $C\left(S_{j}\right)$ represent the area that sensor $S_{j}$ is capable of monitoring. Then, the total energy available for monitoring each location $(x, y)$ can be expressed as:

$$
E_{\text {total }}(x, y)=\sum_{S_{j}: x, y \in C\left(S_{j}\right)} E\left(S_{j}\right)
$$

The contribution of one sensor $j$ to the monitoring task of a particular location can be expressed as a ratio of the sensor's energy and the total energy for that location. Therefore, the overall value of a sensor is taken as the maximum of that sensor's contributions to each location that covered by that sensor:

$$
V\left(S_{j}\right)=\max \frac{E\left(S_{j}\right)}{E_{\text {total }}(x, y)} \quad(x, y) \in C\left(S_{j}\right)
$$

However, this routing metric can perform poorly in some situations. For example, there can be two sensors that have the same value functions defined by Equation (2), but one sensor has much higher residual energy than the other. In this situation, it is clear that the sensor with the higher residual energy is preferred to be elected as active, but the metric defined by Equation (2) will not recognize this. Therefore, the final application cost has been defined as the ratio of the value function and the sensor's residual energy:

$$
\begin{aligned}
C_{a a}\left(S_{j}\right) & =\frac{V\left(S_{j}\right)}{E\left(S_{j}\right)} \\
& =\max \frac{1}{E_{\text {total }}(x, y)} \quad(x, y) \in C\left(S_{j}\right)
\end{aligned}
$$

This application cost can be used as a routing metric. For any two sensors, the link cost between them is expressed as:

$C_{\text {link }}\left(S_{i}, S_{j}\right)=C_{a a}\left(S_{i}\right) \cdot E_{t}+C_{a a}\left(S_{j}\right) \cdot E_{r}$

where $E_{t}$ and $E_{r}$ are the energies required by the sensor to transmit and receive a packet, given respectively as:

$E_{t}=p \cdot\left(E_{- \text {elec }}+e_{-f s} \cdot d^{K}\right)$

$E_{t}=p \cdot E_{\text {elec }}$

$E_{\text {elec }}$ and $e_{f s}$ are the parameters of transmission/reception circuitry and their values are given in Table 1 for the simulations described in this paper, and $d$ is the distance between the nodes. $p$ is the size of the packet, and $K$ is the path loss exponent.

Then, for every sensor the route to the sink is found as the minimum cumulative path's cost:

$C_{a c t}\left(S_{s r c}\right)=\sum_{\left(S_{i}, S_{j}\right) \in p\left(S_{s r c}, S_{d s t}\right)} C_{\text {link }}\left(S_{i}, S_{j}\right)$

where $p\left(S_{s r c}, S_{d s t}\right)$ is the set of links from the source node to the sink along the minimum cumulative path.

Based on the cumulative costs, the protocol determines which sensors should be selected as active, so that the required monitored area is fully covered with the minimum cost. In every round, every sensor assign itself an activation delay proportional to the cost of its route to the base station, and it may announce to its neighbor that it will become active for the incoming round. If, after the activation delay, a sensor finds out that its coverage area is already covered by its 
neighbors, it remains inactive for the incoming round ${ }^{1}$. To achieve the required coverage using nodes with the smallest costs, the sensors with the highest costs have the priority to decide whether they should be active or stay inactive for the incoming round. The detailed implementation of the protocol can be found in [1].

In traditional energy-aware routing [16], the willingness of every sensor to route data is expressed through a cost defined as the inverse value of its residual battery energy:

$$
C_{e a}\left(S_{j}\right)=\frac{1}{E\left(S_{j}\right)}
$$

Based on this routing cost, the sensors with a small amount of residual energy are less likely to be elected as routing stations for data toward the main processing center. However, this routing cost does not consider the importance of particular sensors for the sensing application, in particular, for coverage preservation over the monitored region. This leads to a situation that some sensors, that have fewer neighbors and therefore have the important role of remaining active to sense the environment more frequently, can be still unnecessarily elected as data routers. This can happen especially frequently in the early stages of the network's lifetime. The outcome of this is the premature loss of those sensors, and loss of coverage of parts of the network.

\section{IV.B Application-aware routing in video-based wireless networks}

For an application of video-based wireless sensor networks described in Section II, we must re-define application aware routing. We again assume that the same set of nodes defined previously as $S=\left\{S_{I} \ldots S_{n}\right\}$ is deployed at random locations in the rectangular area $\mathrm{A}$ on the plane $\pi$ shown in Figure 1. The physical location of every node on the plane $\pi$ is presented by coordinates $(x, y)$ and the points of the scene plane $\pi_{1}$ are marked as $\left(x_{c}, y_{c}\right)$. Every location $\left(x_{c}, y_{c}\right)$ on the plane $\pi_{1}$ is characterized by the total energy:

$$
E_{\text {total }}\left(x_{c}, y_{c}\right)=\sum_{S_{j}: x_{c}, y_{c} \in C\left(S_{j}\right)} E\left(S_{j}\right)
$$

where $S_{j}$ is the camera-node and $C\left(S_{j}\right)$ is the intersection of $S_{j}$ 's FoV and $\pi_{1}$.

Following the same logic as described in Section IV.A, we define the final application cost:

$$
C_{a a}\left(S_{j}\right)=\max \frac{1}{E_{\text {total }}\left(x_{c}, y_{c}\right)} \quad\left(x_{c}, y_{c}\right) \in C\left(S_{j}\right)
$$

Note that up to now, we have defined the application cost using the coordinates $\left(x_{c}, y_{c}\right)$ on the scene plane $\pi_{1}$. However, the cost of a link between the nodes will depend on the physical positions of the nodes in plane $\pi$, assuming that $E_{t}$ is a function of the distance between the nodes.

\footnotetext{
1 In order to find out their state in the incoming round, the sensors create a grid within their sensing ranges, and point-by-point they decide if their sensing ranges are covered by neighboring nodes. However, as stated in [1] any coverage preserving rule can be used here.
}

Therefore, the total routing cost for every camera-node, expressed as a cumulative function of link costs among the nodes, is a function of the nodes' locations on plane $\pi$ :

$$
C_{a c t}\left(S_{s r c}\right)=\sum_{\left(S_{i}, S_{j}\right) \in p\left(S_{s r c}, S_{d s t}\right)} C_{\text {link }}\left(S_{i}, S_{j}\right)
$$

At the beginning of every round, the coordinates of the "user request window" are randomly chosen within the area $A$. For a requested area bounded by the user request window, all cameras that cover that part of the scene are determined. The set of active camera-nodes that cover the requested part of the scene with the minimum cost is found by taking into consideration the cumulative costs defined by Equation (11). The same procedure from Section IV.A is followed here in order to find the set of active camera-nodes.

At the beginning, all nodes are in the active state. The nodes with higher total routing cost have priority to decide if they will remain in the active state, or they will turn off. The decision is made based on whether all points covered by the camera-node's FoV are covered by other nodes' FoVs with lower cumulative cost.

\section{Comparison of an Application-aware Protocol in Wireless Sensor Networks and Video-based Sensor Networks}

In this section we present the results of simulations when the modified application-aware protocol described in IV.B is applied to video-based networks, for the case when the system serves one user. Table 1 shows the parameters used for the simulations. We measured the time for which the coverage of the monitored area drops to a certain percentage. These simulations are repeated for the case of an applicationaware routing protocol in a traditional sensor network, as described in IV.A. The results for both the traditional sensor network and the video-based sensor network are compared with the case when an energy-aware routing protocol is used. All simulations can be easily modified for the scenario where the video-based network serves a large number of users at the same time, and requests to the system in every time instance require almost complete coverage of the monitored area.

\section{V.A Application-aware routing in video-based networks}

As already shown in [1], application-aware routing achieves significant improvement in coverage time, which is the time during which the network is able to preserve the full coverage of the monitored area, over energy-aware routing for traditional sensor networks, as shown in Figure 2. However, in video-based networks, this application-aware routing protocol does not preserve the coverage for a longer time compared to energy-aware routing. The results of simulations for video-based networks are shown in Figure 3. Routing based on the energy-aware cost unexpectedly performs better for video-based networks. This leads us to believe that this application-aware protocol, which was designed for traditional wireless sensor networks, is not completely 
applicable to the coverage preserving task in video-based networks.

The reason for this result lies in the mismatch between the cameras' physical positions and the cameras' FoVs. For the sake of explanation, we examine the case when a user requests to see a part of the scene on scene plane $\pi_{1}$, as shown in the Figure 1. The camera-nodes that monitor the requested part can be located anywhere on the mounting plane $\pi$. Among all the possible camera-nodes that monitor the area of interest, the application-aware algorithm selects the minimum set of camera-nodes with the smallest cumulative cost. A set of active nodes that cover the part of the area for a minimum cost is chosen from a set of camera-nodes placed at random locations in the network plane $\pi$. In the case of traditional wireless sensor networks, the requested part of the scene determines the locations of all sensors that take part in coverage of that part of scene. In order to preserve coverage, the distance between any two neighboring active nodes can be at most twice the sensing range, which means that the active nodes are grouped together, which is not the case for videobased sensor networks. In application-aware routing, the cost of a node is a function of the available energy of the node, and also of other nodes whose FoVs (sensing ranges) overlap with the node's FoV. In the case of a traditional sensor network, this cost function tells us how redundantly a sensor is covered, but also evaluates the sensor from the routing perspective. For example, a sensor with low cost is usually a sensor deployed in a dense area, surrounded by many nodes that are equally important as routers and which redundantly cover its sensing area. Therefore, the loss of this sensor will not influence the coverage, nor will it mean the loss of important relaying nodes.

In video-based networks, however, this cost function values the node's importance only from the coverage perspective. Although this cost function selects as active nodes the nodes that are more redundantly covered, this selection does not take into consideration the node's role as a potential router. For example, it can happen that a cameranode is located in a scarcely-deployed area, so that it is far away from its closest neighbors, but its FoV is overlapped

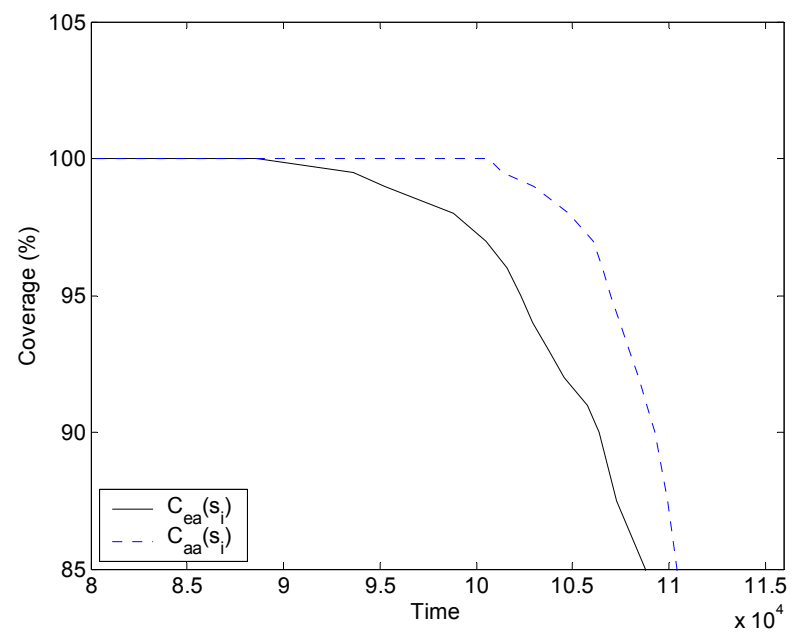

Figure 2 Traditional wireless sensor network- coverage over time. $\mathrm{C}_{\mathrm{ea}}$ is energy-aware cost and $\mathrm{C}_{\mathrm{aa}}$ is application-aware cost. with the FoVs of several other cameras. In an early stage of the network, this camera-node can have an important role as a router, and its energy should not be spent on the sensing task. However, because its FoV is already redundantly covered with that of many other cameras', its cost according to Equation 10 will be relatively small, which makes it suitable for selection as an active camera for the coverage task.

Among all nodes that cover the requested part of the scene, the application-aware protocol selects those nodes that have the smallest total cumulative path cost - a sum of all the links' costs from the node to the sink. On other hand, it is well known that nodes close to the base station are frequently used as routers of data from the other nodes toward the base station and therefore loose their energy much faster compared to the nodes in the rest of the network However, it is still possible that their FoVs are redundantly covered with the FoVs of other cameras throughout the network, which makes their cost relatively small. Because they are closer to the base station, their total cumulative path cost is in many cases smaller then that of nodes further away from the base station. This makes them suitable for selection as active sensing nodes very frequently. As a result, the loss of these important routers is unavoidable. This speeds up the loss of energy of the rest of the network and makes the "hotspot" problem worse.

Therefore, although application-aware routing selects the nodes in the right manner from the coverage perspective, it overlooks the fact that the cameras' FoVs are displaced relative to the camera locations. Thus, when used in a network equipped with cameras, application-aware routing makes energy-inefficient selection of nodes, which leads to loss of a large number of nodes in the early stages of the network operation.

In video-based networks, the energy-aware routing cost surprisingly outperforms the application-aware routing cost, in coverage-time. This cost function does not measure the particular node's importance to the coverage application, it only determines the node's ability to be active, based solely on the node's energy. Although this cost function does not have control over the coverage directly, the coverage is maintained for a longer time thanks to two factors: the more

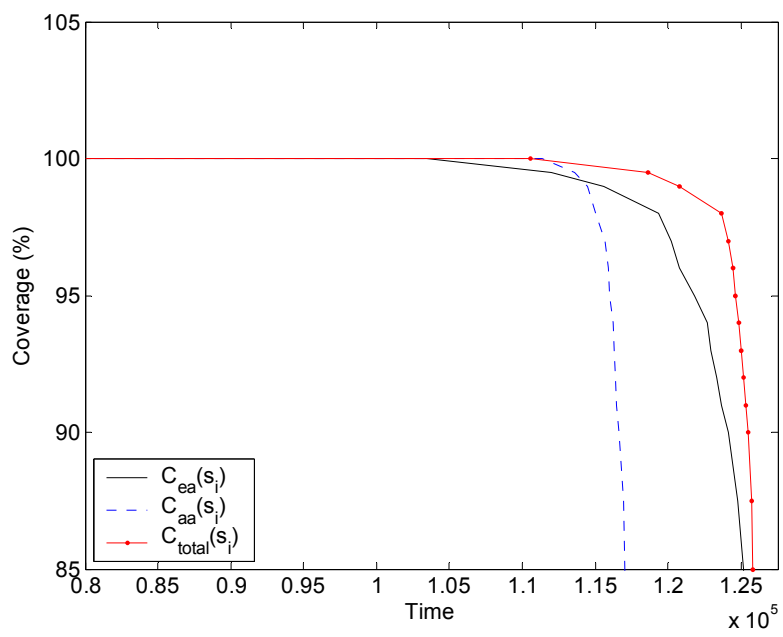

Figure 3 Video-based sensor network- coverage over time. $\mathrm{C}_{\mathrm{ea}}$ is energyaware cost, $\mathrm{C}_{\mathrm{aa}}$ is application-aware cost and $\mathrm{C}_{\text {total }}$ is total cost. 
balanced energy spent among the nodes and the uncontrolled positions of the cameras' FoVs over the monitored area. A node will be selected as an active node if it has more energy than the other potential active nodes, which directly prolongs the lifetime of every node. Over time, the nodes at random locations die across the area, and not necessarily close to the sink, as in the case of the application-aware cost. Due to the unpredicted positions of the cameras' FoVs, the lost coverage due to the death of nodes will be also more or less randomly distributed across the area.

Also, it is interesting to notice that energy-aware routing not only outperforms application-aware routing in the time during which the coverage is preserved, but it also gives, for the same simulation parameters, the longer coverage-time in the case when it is used in video-based networks, than in the traditional sensor networks. This result can also be explained as a consequence of the uncontrolled positions of the cameras' FoVs, and the fact that in each round the active camera-nodes are chosen from a set of nodes that are dispersed over the whole area. This allows the algorithm to choose among nodes with different routing and coverage capabilities, which in turn leads to even more balanced energy spending and more consistent coverage preservation than in the case of traditional sensor networks.

\section{$V . B$ Combined application and routing cost}

The simulation results from the previous subsection indicate that the problem of application-aware routing in video-based networks is hard to manage in an integrated manner. The results point out that every camera-node should be validated by two separate costs - coverage cost and routing cost. The first cost is related to how important the camera is for covering some part of the monitored area, and the second cost evaluates the importance of the node to act as a possible router of data toward the base station, with the goal of achieving more balanced energy spending over the network. The total cost for every camera-node can be expressed as the sum of these two cost functions, given as:

$$
\begin{aligned}
C_{\text {total }}\left(S_{j}\right) & =C_{e a}\left(S_{j}\right)+C_{a a}\left(S_{j}\right) \quad\left(x_{c}, y_{c}\right) \in C\left(S_{j}\right) \\
& =\frac{1}{E\left(S_{j}\right)}+\max \frac{1}{E_{\text {total }}\left(x_{c}, y_{c}\right)}
\end{aligned}
$$

This combined cost function reduces the problem that exists with the application-aware cost, where important routing nodes can often be selected as active sensing nodes. The coverage time for the total cost function is shown in Figure 3. The total cost function, when used for the same simulation scenario, leads to prolonged time during which the coverage of the monitored area is preserved. This cost function also reduces the energy consumption of the nodes compared to the application aware routing cost, as shown in Table 2. Therefore, the total cost function combines the best attributes of both application-aware and energy-aware cost functions.
With a change in density of camera-nodes in the network, we can observe the same relationships between all three protocols described in this section. As an illustration, in Figure 4 we show the results of simulations for all three protocols when we change the number of camera-nodes in the area and measure the time until less than $95 \%$ of the total area is being covered by cameras. It is noticeable that in all cases the total cost function shows slightly better results than the other two cost functions.



Figure 4 . The time at which $95 \%$ of the total monitored area is covered, for different number of camera nodes in the video-based sensor network

\begin{tabular}{|l|l|}
\hline Parameter & Value \\
\hline Size of the network & 100 X $100 \mathrm{~m}$ \\
\hline Size of monitored scene & $100 \times 100 \mathrm{~m}$ \\
\hline Bit rate & $500 \mathrm{bits} / \mathrm{s}$ \\
\hline Number of nodes & 150 \\
\hline Initial energy & $2 \mathrm{~J}$ \\
\hline $\mathrm{E}_{\text {elec }}$ & $50 \mathrm{~nJ} / \mathrm{bit}$ \\
\hline $\mathrm{e}_{\mathrm{fs}}$ & $100 \mathrm{pJ} / \mathrm{bit} / \mathrm{m}^{2}$ \\
\hline $\mathrm{k}$ & 2 \\
\hline sensing range & $15 \mathrm{~m}$ \\
\hline Camera's FoV radius & $15 \mathrm{~m}$ \\
\hline
\end{tabular}

Table 1 - Simulation parameters.

\begin{tabular}{|c|c|c|c|}
\hline & $\mathrm{Cea}(\mathrm{Si})$ & $\mathrm{Capp}(\mathrm{Si})$ & Ctotal(Si) \\
\hline $\begin{array}{c}\text { Average spent } \\
\text { power per path } \\
(\mathrm{mW})\end{array}$ & 0.1091 & 0.1251 & 0.1121 \\
\hline
\end{tabular}

Table 2 Comparison of average spent power per one path for different routing costs, for 150 camera-nodes in the network.

\section{Conclusion and Future Work}

In this work, we analyzed the results obtained when an application-aware protocol, which integrates the routing and coverage preserving application task for traditional wireless sensor networks, is applied to video-based sensor networks. Our results, which can influence future directions in the 
development of video-based sensor networks, show that the coverage problem in video-based sensor networks cannot be treated in the same way as in traditional wireless sensor networks. The simulation results were analyzed thoroughly, and we pointed out the main differences between traditional sensor networks and video-based sensor networks, from the perspective of the coverage preservation problem.

We analyzed the situation when the camera-nodes monitor the scene in one plane, and we found that, due to the unpredictable positions of the cameras' FoVs, the applicationaware routing protocol behaves intrinsically different than for the case when it is applied to traditional sensor networks. We found that the coverage and routing problems exist in videobased networks as two separate problems. As a further step, we introduced the total cost that combines the coverage and routing costs for each camera-node. This cost function, when used with routing over the minimum cumulative cost path, achieves a slight improvement over energy-aware routing, but this is sufficient to show the direction in which this problem should be further studied.

We are aware of some simplifications which we used in this work, and in the future our goal will be to further investigate this problem with more realistic models. For example, we want to analyze three-dimensional coverage and to take into consideration the collaboration among the cameras in order to reconstruct the image of some monitored part of scene. Also, we want to consider the ability of the cameras to obtain the information about the scene with different resolution. We will also investigate the same problem for the case of multi-modal networks - networks that combine video cameras with other types of sensors (acoustic, for example).

\section{Acknowledgement}

The authors would like to thank to Mark Perillo for his help with this work. This research was supported by NSF ECS 0428157.

\section{References}

[1] M. Perillo, W. Heinzelman, "DAPR: A Protocol for Wireless Sensor Networks Utilizing an Application-based Routing Cost", In Proceedings of the IEEE Wireless Communications and Networking Conference (WCNC '04), March 2004.

[2] U.M. Erdem, S. Sclaroff, "Optimal Placement of Cameras in Floorplans to Satisfy Task Requirements and Cost Constraints", In Proceedings of Omnivis2004, The fifth Workshop on Omnidirectional Vision, Camera Networks and Non-classical cameras, May 2004

[3] R. Holman, J. Stanley, T.Ozkan-Haller, “Applying Video Sensor Networks to Nearshore Environment Monitoring," IEEE Pervasive Computing, vol 2, no. 4, 2003, pp. 14-21

[4] K. Obraczka, R. Manduchi, J.J. Garcia-Luna-Aveces, "Managing the Information Flow in Visual Sensor Networks", In Proceedings of the 5th International Symposium on Wireless Personal Multimedia Communications, October 2002

[5] W. Feng, B. Code, E. Kaiser, M. Shea, W. Feng, L. Bavoli, "Panoptes: Scalable Low Power Video Sensor Networking Technologies", ACM Transactions on Multimedia Computing, Communications and Applications, January 2005

[6] P. Kulkarni, D. Ganesan, P. Shenoy, "The Case for Multi-tier Camera Sensor Netwoks", In Proceedings of the International Workshop on Network and Operating Systems Support for Digital Audio and Video (NOSSDAV), 2005

[7] M. Chu, J. Reich, F. Zhao, "Distributed Attention in Large Scale Video Sensor Networks", Intelligent Distributed Surveillance Systems 2004 seminar; February 2004.

[8] L. Jiao, Y.Wu, g.Wu, E.Y.Chang, Y.-F. Wang, "The Anatomy of a Multi-Camera Video Surveillance System" ACM Multimedia System Journal Special Issue, vol. 10, no 2, October 2004

[9] D. Devarajan, R. Radke, "Distributed Metric of Large Camera Networks", In Proceedings of the First Workshop on Broadband Advanced Sensor Networks, October, 2004,

[10] F. Ye, G. ,Zhong, J. Cheng, S. Lu, L. Zhang, "PEAS: A Robust Energy Conserving Protocol for Long Lived Sensor Networks", In Proceedngs of Twenty Third International Conference on Distributed Computing Systems, 2003

[11] Z. Zhou, S. Das, G. Gupta, "Variable Radii Connected Sensor Cover in Sensor Networks", In Proceedings of the IEEE International Conference on Sensor and Ad Hoc Communications and Networks (SECON), Oct 2004.

[12] S. Shakkottai, R. Srikant, N. Shroff, "Unreliable Senror Grids: Coverage, Connectivity and Diameter", In Proceedings of INFOCOM 2003, April 2003.

[13] X. Wang, G. Xing, Y. Zhang, C. Lu, R. Pless, C.D.Gill, "Integrated Coverage and Connectivity Configuration in Wireless Sensor Networks" In Proceedings of the First International Conference on Embedded networked sensor systems, 2003.

[14] D. Tian, N. Georganas, "A Node Scheduling Scheme for Energy Conservation in Large Wireless Sensor Networks", Wireless Communications and Mobile Computing Journal, vol.3, no. 2, pp. 271-290, March 2003.

[15] M. Marengoni, B.A. Draper, A. Hanson, R. Sitaraman, "A System to Place Observers on a Polyhedral Terrain in Polynomial Time", Image and Vision Computing, vol. 18, pp773-780, 1996.

[16] C.-K. Toh, "Maximum Battery Life Routing to Support Ubiquitous Mobile Computing in Wireless Ad-Hoc Networks", IEEE Communications Magazine, vol. 39, no. 6, June 2001.

[17] http://telepresence.dmem.strath.ac.uk

[18] C. Huang, Y.Tseng, L. Lo, "The Coverage Problem in Three Dimensional Wireless Sensor Networks", In proceedings of IEEE Globecom, 2004 